\title{
SESELEH WANGUN INTRINSIK LAN BASITA PARIBASA RING PUPULAN SATUA BAWAK KACUNDUK RING BESAKIH PIKARDIN I GUSTI GEDE DJELANTIK SANTHA
}

\author{
Ni Md. Utari Rahayu ${ }^{1}$, I. A. Pt. Purnami ${ }^{1}$, I Kt. Paramarta ${ }^{2}$ \\ Jurusan Pendidikan Bahasa Bali \\ Universitas Pendidikan Ganesha \\ Singaraja, Indonesia \\ e-mail: \{rahayu66@rocketmail.com, dayupurnamiku@yahoo.co.id, \\ ketutgembel@yahoo.co.id\}@undiksha.ac.id
}

\begin{abstract}
Kuub
Tetilik puniki matatujon nelatarang indik: (1) wangun intrinsik, lan (2) basita paribasa sane wenten ring pupulan satua bawak Kacunduk ring Besakih pikardin I Gusti Gede Djelantik Santha. Jejering tetilik inggih punika pupulan satua bawak Kacunduk ring Besakih pikardin I Gusti Gede Djelantik Santha. Panandang tetilik inggih punika wangun intrinsik lan basita paribasa. Tetilikan puniki ngranjing ring tetilikan deskriptif kualitatif. Kramaning mupulang data ring tetilikan puniki nganggen kramaning dokumentasi lan nganggen piranti kartu data. Data tureksa sane kaanggen: (1) nyelehin data, (2) nelatarang data, lan (3) pamicutet. Pikolih tetilik pupulan satua bawak Kacunduk ring Besakih pikardin I Gusti Gede Djelantik Santha, sakadi: (1) wangun intrinsik wenten pitu, sakadi: unteng, lelintihan, pragina, rerawatan, genah linggih sang pengawi, paribasa lan piteket. (2) basita paribasa kapolihang 9 soroh sakadi: bebladbadan, rawos ngempelin, sesawangan, sesimbing, sesenggakan, sesonggan, wewangsalan, tetingkesan, lan sesawen.
\end{abstract}

Kruna jejaton: Basita paribasa, Satua bawak, Wangun intrinsik

Abstrak

Penelitian ini bertujuan untuk menjelaskan: (1) unsur intrinsik, dan (2) basita paribasa dalam kumpulan cerpen Kacunduk ring Besakih karya I Gusti Gede Djelantik Santha. Subjek penelitian adalah kumpulan cerpen Kacunduk ring Besakih karya I Gusti Gede Djelantik Santha. Objek penelitian adalah unsur intrinsik dan basita paribasa. Penelitian ini masuk ke dalam penelitian deskriptif kualitatif. Metode pengumpulan data dalam penelitian ini menggunakan metode dokumentasi serta menggunakan sarana kartu data. Analisis data yang digunakan: (1) identifikasi data, (2) menyajikan data, dan (3) kesimpulan. Hasil penelitian kumpulan cerpen Kacunduk ring Besakih karya I Gusti Gede Djelantik Santha, antara lain: (1) unsur intrinsik ada tujuh, seperti: tema, alur, penokohan, latar, sudut pandang, gaya bahasa dan amanat, (2) basita paribasa ditemukan 9 jenis, seperti: bebladbadan, rawos ngempelin, sesawangan, sesimbing, sesenggakan, sesonggan, wewangsalan, tetingkesan, dan sesawen.

Kata kunci: Basita Paribasa, Cerpen, Unsur Intrinsik 


\section{Abstract}

This study aims to explain: (1) intrinsic elements, and (2) basita paribasa in a collection of short stories Kacunduk ring Besakih by I Gusti Gede Djelantik Santha. The subject is a collection of short stories Kacunduk ring Besakih by I Gusti Gede Djelantik Santha. The object is intrinsic element and basita paribasa. This research goes into qualitative descriptive research. Methods of data collection in this study using the method of documentation and using the means of data cards. Analysis of data used: (1) identification of data, (2) present data, and (3) conclusion. The result of the study of the cluster of Kacunduk ring Besakih by I Gusti Gede Djelantik Santha, among others: (1) intrinsic elements there are seven, such as: theme, plot, character, background, point of view, style and mandate, (2) basita paribasa found 9 Types, such as: bebladbadan, rawos ngempelin, sesawangan, sesimbing, sesenggakan, sesonggan, wewangsalan, tetingkesan, and sesawen.

Keywords: Basita Paribasa, Intrinsic Element, Short story

\section{PURWAKA}

Kasusastraan inggih punika kria sastra sane kakawi olih para sujana sane madaging kawentenan-kawentenan ring sajeroning kauripan tur kasurat nganggen basa pinaka sarana anggen nelatarang. Manut Gautama (2007: 2) Kesusastraan inggih punika makesamian kaweruhan sane madasar antuk pikenoh sane prasida kasinahang antuk tulisan sane becik tur ngalangunin. Kasusastraan manut aabnyane wenten kabaos kasusastraan Bali purwa lan kasusastraan Bali anyar. Sane kabaos kesusastraan Bali purwa inggih punika kasusastraan sane sampun wenten saking pinih rihin ring Bali. Sane kabaos kasusastraan Bali anyar inggih punika kria sastra sane sampun nglimbak lan wangunnyane pateh sakadi kasusastraan Indonesia. Wangun utawi wentuk lan daging kesusastraan Bali anyar makeh polih iusan saking kesusastraan Indonesia lan kasusastraan barat utawi asing. Kasusastraan Bali anyar punika kria sastra sane kawentenannyane ritatkala krama Bali sampun keni modernisasi.

Kasusastraan Bali anyar sampun kakawitin saking warsa 1931 mawentuk novel sane kawijilang olih Balai Pustaka sane mamurda Nemoe Karma pikardin I Wayan Gobiah. Nanging Putra (2010: 15) maosang sadurung medalnyane novel Nemoe Karma, ring warsa 1910-an sampun medal kria sastra Bali anyar marupa cerpen sane kakawi olih I Made Pasek lan Mas Nitisastro. Raris cerpen sane kakawi olih I Made Pasek miwah Mas Nitisastro inucap kamanggehang pinaka wit ring panglimbak sastra Bali anyar. Kasusastraan Bali anyar punika wenten petang pahan minakadi puisi, satua bawak, drama miwah novel. Silih tunggil kria sastra sane jagi katilikin olih panilik inggih punika satua bawak (cerpen).

Satua bawak inggih punika silih tunggil kria sastra sane ngranjing ring prosa Bali anyar. Satua bawak inggih punika sasorohan prosa Bali anyar sane wangunnyane alit taler yening saihang tekening novel utawi roman, satua bawak (cerpen) puniki caritanyane pinih bawak (Tarigan, 1984: 164). Sakadi wastannyane, satua bawak punika wantah carita sane bawak. Satua bawak inggih punika silih sinunggil kria sastra sane madaging kirang langkung mawit saking 500 kruna nyantos 10.000 kruna. Satua bawak kawangun antuk kalih wangun inggih punika wangun intrinsik lan wangun ekstrinsik.

Wangun intrinsik kaanggen natasang lan nlatarang kawigunan ring sajeroning paiketan unsur fiksi sane nguningayang indik daging kria sastra. Wangun intrinsik prasida ngawinang sang pangwacen dangan ngaresepang sapulah-palih daging carita satua bawak. Saduaning wangun intrinsik punika sane ngwangun kria sastra 
mawit saking jero utawi tengah. Wangun intrinsik ring satua bawak minakadi (unteng, lelintihan carita, pragina, rerawatan, genah linggih sang pangawi, paribasa lan piteket). Lelintihan punika prasida kaanggen dasar olih panilik ri kala nyelehin daging-daging satua bawak sane jagi katilikin. Punika taler wangun ekstrinsik inggih punika samian wangun sane ngwangun kria sastra ring jaba utawi ring sisi kria sastra minakadi para pangwacen, embasnyane sang pangawi, pengalaman urip pangawi, kulit buku, tebel buku, nanging prasida ngiusin kria sastranyane.

Kria sastra anyar utamanyane satua bawak ketah pastika katelatarang lan kasobyahang nganggen basa mangda kadadosang carita lan prasida karesepang. Warsito (2012: 73) maosang basa pinaka piranti mabebaosan miwah piranti anggen nyihnayang rasa taler pikayunan, sane prasida kasobiahang antuk sasuratan, lisan, miwah pisarat. Lianan tekening basa sadina-dina sane dangan karesepang, kria sastra utamannyane satua bawak taler madaging panglengut basa. Kawentenan basa-basa sane lengut punika pastika mapaiketan sareng paribasa. Paribasa ring sajeroning basa Bali kabaos basita paribasa. Tinggen (1988: 7) maosang, basita paribasa pinaka sinalih tunggil wangun kebudayaan Bali, sane madaging guna sarat luhur taler madue iusan ring dasar kauripan parajana, sane nganggen basita paribasa ri tatkala mabebaosan. Taler Simpen (1980: 5) maosang, basita paribasa pinaka basa rinengga sane dados rerasmen wiadin panglengut basa, rikala mababaosan kalih magegonjakan anggen pasimakraman.

Makudang-kudang para sujana madue panampen sane malianan indik soroh-soroh basita paribasa. Silih sinunggil panampen sane jagi kaanggen dasar tatepasan olih panilik inggih punika panampen saking Tinggen (1988: 6) nyorohang basita paribasa antuk telulas soroh inggih punika cecimpedan, bladbadan, rawos ngempelin, sesawangan, sesimbing, sloka, sesenggakan, sesonggan, sesapaan, wewangsalan, peparikan, tetingkesan lan sesawen. Kawentenan basita paribasa ring aab sakadi mangkin kapanggih ring paplajahan bahasa Bali ring sekolah-sekolah. Kawentenan ring paplajahan bahasa Bali punika basita paribasa wantah kapanggih nganggen wentuk-wentuk lengkara sane kadadosang imba ring buku teks para sisiane. Imba-imba lengkara punika pastika meweh karesepang olih sisia-sisiane sane ngwacen mawinan imba-imba punika wantah mawentuk lengkara kemanten. Sang sane ngwacen pastika nenten uning ri kala napi iraga nganggen basita paribasa punika ring sajeroning mabebaosan sadinadina. Kawentenan basita-paribasa pastika dangan karesepang yening wentuknyane nenten marupa lengkara kemanten nanging kaadungang ring sajeroning bebaosan. Bebaosan-bebaosan sane madaging basita paribasa makeh kacingak ring sajeroning kria sastra.

Kawentenan basita paribasa ring kria sastra makeh kawentenannyane sakadi ring naskah drama, novel, puisi lan satua bawak sane kasinahang saking bebaosanbebaosan pragina ring carita. Nanging, yening selehin malih kari arang kapanggihin basita paribasa ring sajeroning satua bawak. Mawinan kadi asapunika panilik pacang nilikin silih tunggil buku pupulan satua bawak pikardin I Gusti Gede Djelantik Santha sane mamurda "Kacunduk ring Besakih". Pupulan satua bawak sane mamurda Kacunduk ring Besakih puniki madaging 15 murda satua bawak. Saking 15 murda satua bawak punika, panilik ngambil 5 murda satua bawak luire Alpaka Nilar Sentana, Jatu Karma, Kehkeh Siap, Nyelsel Raga, lan Pager Neda Tetanduran. Panilik wantah nilikin murda-murda punika mawinan saking limolas murda wantah lalima sane makeh madaging basita paribasa. Antuk punika panilik pacang nglaksanayang tetilikan sane mamurda, "Seseleh Wangun Intrinsik lan Basita Paribasa ring Pupulan Satua Bawak Kacunduk ring Besakih Pikardin I Gusti Gede Djelantik Santha".

Bantang pikobet tetilik: (1) sapunapi wangun intrinsik sane wenten ring Pupulan Satua Bawak Kacunduk ring Besakih pikardin I Gusti Gede Djelantik Santha?, (2) sapunapi kawentenan basita paribasa sane wenten ring Pupulan Satua Bawak Kacunduk ring Besakih pikardin I Gusti Gede Djelantik Santha? 
Tejujon tetilik: (1) mangda prasida nelatarang wangun intrinsik sane wenten ring Pupulan Satua Bawak Kacunduk ring Besakih pikardin I Gusti Gede Djelantik Santha, (2) mangda prasida nelatarang kawentenan basita paribasa sane wenten ring Pupulan Satua Bawak Kacunduk ring Besakih pikardin I Gusti Gede Djelantik Santha.

Kawigunan pamucuk sane prasida kapolihang mawiguna kanggen nglimbakang widya pangweruhan sastra lan basa. Kaaptiang mangda tetilikan puniki prasida nglimbakang dasar tetimbang ngenenin indik wangun intrinsik lan basita paribasa ring satua bawak mabasa Bali sumangdene prasida sayan-sayan nglimbak. Kawigunan panglimbak sane kapolihang: (1) majeng ring mahasisia, mangda prasida kanggen pratiwimba lan sesuluh yening jagi ngamargiang tetilikan asoroh sane mapaiketan ring tetilikan puniki, (2) majeng ring para jana, mangda tetilikan puniki prasida dados sesuluh ring kauripan majalaran antuk ngresepin pabesen utawi tetuek sane wenten ring pupulan satua bawak Kacunduk ring Besakih, (3) majeng ring pangwacen, mangda prasida ngicen pangresep ajahajahan/pangweruhan indik wangun intrinsik lan basita paribasa ring pupulan satua bawak Kacunduk ring Besakih, (4) majeng ring panilik, prasida kaanggen pengalaman sapunapi indik sapulah-palih nureksain utawi nyelehin wangun intrinsik lan kawentenan basita paribasa taler sorohsorohnyane sane wenten ring kria sastra.

Arikunto (2005: 39) maosang, kawentenan sepat siku-siku dahat mabuat pisan saduaning prasida ngicenin pamargi lan ngwantu panilik muputang tetilikannyane. Sepat siku-siku puniki ngicenin gambaran/gaguwet indik teori-teori sane jagi kaanggen dasar ri kalaning ngamargiang tetilikan. Sepat siku-siku sane kawedarang mangdane madue paiketan sareng dadalan pikobet sane sampun kakaryanin.Sepat siku-siku sane kaanggen, sakadi: (1) kasusastraan Bali, (2) satua Bawak, (3) wangun Kria Sastra lan, (4) basita Paribasa.

Kasusastraan inggih punika pikolih saking hredaya sang pangawi sane kametuang antuk basa. Kasusastraan mawit saking basa Sansekerta sane linggan ipun 'sastra'. Kruna sastra mawit saking kruna 'sas' miwah 'tra'. 'Sas' maartos kaweruhan, 'tra' maartos piranti sane kaanggen. Dadosnyane kasusastraan inggih punika kaweruhan sane becik sane metu saking manah pikayun para pangawi ring Bali sane dados piranti sasuratannyane antuk aksara Bali miwah aksara latin. Merta dkk. (2002: 69) maosang "kasusastraan inggih punika palaning reriptan sang kawiswara malarapan antuk pawisik/wahyu sane kaiket antuk basa lan aksara sane lengut".

Suroto (1989: 3) maosang satua bawak inggih punika silih sinunggil karya sastra sane marupa gancaran, inggih punika "kria sastra sane nelatarang indik pikobet sane mapalet-paletan. Satua bawak inggih punika kakawian gancaran sane madaging carita kauripan manusa majalaran antuk pragina ring jeroning carita punika. Putera (2006: 6) maosang watesan satu bawak inggih punika, akeh kruna 500 nyaantos 10.000 kruna utawi 2 nyantos 25 kaca.

Soang-soang wangun kria sastra pastika madue wangun sane maendahan. Wangun ring sajeroning kria sastra pinih mautama pisan krana wangun puniki pinaka dasar kaanggen ri kala makarya kria sastra minakadi satua bawak. Wangun kria sastra inggih punika paiketan ring makasamian pah-pahan kria sastra sane mawentuk makudang-kudang wangun sane kasusun kadadosang siki tur ngamedalang sikian unteng sane prasida ngawetuang pangarsa ring sang sane ngwacen. Kria sastra minakadi satua bawak nyaritayang pidabdab ring para jana sane kasinahang nganggen kruna-kruna mangda dados sikian lengkara sane becik tur ngulangunin. Manut Wellek \& Warren (ring Karmini, 2011: 14) akeh sane kabaosang indik kritikus ri kala nilikin indik kria sastra, minakadi wangun intrinsik lan ekstrinsik.

Wangun intrinsik inggih punika wangun utawi srana sane ngwangun kria sastra ring tengahing kria sastra, minakadi: (1) unteng, (2) lelintihan carita, (3) pragina, (4) rerawatan, (5) genah linggih sang pangawi, (6) paribasa, lan (7) piteket. Wangun intrinsik prasida ngawinang sang 
pangwacen dangan ngaresepang sapulahpalih daging carita satua bawak. Saduaning wangun intrinsik punika sane ngwangun kria sastra mawit saking jero utawi tengah.

Wangun ekstrinsik inggih punika wangun sane nenten prasida kacingak ring tengahing carita. Nanging mapaiketan sareng carita miwah prasida ngicenin pangweruh ring sajeroning carita miwah prasida ngicenin pangweruh ring sajeroning carita. Sane ngeranjing ring sajeroning wangun ekstrinsik sakadi minakadi kasenengan para pangwacen, embasnyane sang pangawi, pengalaman urip pangawi, kulit buku, lan tebel buku. Yadiastun wangun ekstrinsik wantah ngwangun kria sastra saking jaba utawi sisi nanging, prasida ngiusin kria sastra punika taler tata wangun puniki mapaiketan sareng wangun sane siosan.

Para sujana madue panampen soang-soang indik basita paribasa punika. Simpen (1980: 5) maosang basita paribasa inggih punika basa rinengga, punika dados rerasmen basa wiadin panglengut basa, ri kala mabebaosan kalih magegonjakan, anggen pasimakrama. Taler manut Tinggen (1988: 7) basita paribasa pinaka sinalih tunggil wangun kebudayaan Bali, sane madaging guna sarat- guna sarat luhur taler masue iusan saking dasar kauripan parajana, sane nganggen basita paribasa ri tatkala mabebaosan.

Manut saking panampen ring ajeng, nenten ja banget matiosan. Dadosnyane prasida kacutetang basita paribasa inggih punika rerasmen basa sane kaanggen magegonjakan miwah ngamedalang pikayunan antuk nyaihang kahanan lan laksanan jadma sareng piranti, beburon, tetanduran miwah palemahan sakewanten samian punika madaging guna sarat sane kaanggen sesuluh ngamargiang kahuripan.

Soroh-soroh basita paribasa manut I Nengah Tinggen kasorohang dados telulas soroh sakadi: (1) cecimpedan, (2) bebladbadan, (3) raos ngempelin, (4) sesawangan, (5) sesimbing, (6) sloka, (7) sesenggakan, (8) sesonggan, (9) sesapan, (10) wewangsalan, (11) peparikan, (12) tetingkesan, miwah (13) sesawen.

\section{KRAMANING TETILIK}

Tetilik puniki ngangge tetilik deskriptif kualitatif. Tetilik deskriptif kualitatif nganggen lan ngandelang data sane marupa non verbal (tertulis). Wenten lelima papalih paridabdab kramaning tetilik sakadi: (1) palihan tetilik, palihan tetilik mapaiketan ri kala nglaksanayang tetilik. Palihan tetilik maka dasar kanggen sadurung nglaksanayang tetilik. Palihan tetilik inggih punika strategi ring sajeroning ngatur tetilik mangda penilik prasida ngamolihang data sane patut lan sane manut ring karakteristik variabel lan tetujon tetilik; (2) jejering lan panandang tetilik, jejering tetilik puniki pupulan satua bawak Kacunduk ring Besakih pikardin I Gusti Gede Djelantik Santha, panandang tetilik wangun intrinsik lan basita paribasa; (3) mupulang data, mupulang data, mupulang data pidabdab sane nyinahang sapunapi data kapolihang. Tetilik kualitatif molihang data marupa kruna/lengkara. Kramaning kanggen mupulang data inggih punika kramaning dokumentasi santukan data pupulan satua bawak basa Bali marupa sesuratan (buku); (4) piranti tetilik, piranti sane kanggen mupulang data inggih punika kartu data. Kartu data kanggen nyurat pikolih wangun intrinsik lan basita paribasa; (5) data tureksa, pidabdab sane kalaksanayang sajeroning data tureksa sakadi ngatur, nyusun, nagingin kode, lan nlatarang data. Data sane kapolihang punika kaubah miwah katlatarang antuk lengkara utawi kadadosang bahbahan lengkara-lengkara.

\section{PIKOLIH LAN TETEPASAN}

Pikolih tetilik puniki klaksanayang antuk kramaning tetilik sane marupa kramaning dokumentasi. Pikolih ring kramaning dokumentasi ring tetilikan puniki inggih punika marupa pupulan satua bawak sane mamurda Kacunduk ring Besakih pikardin I Gusti Gede Djelantik Santha. 
Pupulan satua bawak Kacunduk ring Besakih madaging 15 murda satua bawak, nanging panilik wantah nilikin 5 murda satua bawak minakadi Alpaka Nilar Sentana, Jatu Karma, Kehkeh Siap, Nyelsel Raga lan Pager Neda Tetanduran. Pikolih lan tetepasan tetilik wangun intrinsik sajeroning pupulan satua bawak Kacunduk ring Besakih pikardin I Gusti Gede Djelantik Santha katlatarang ring sor puniki.

Satua bawak kapertama sane katilikin inggih punika mamurda Alpaka Nilar Sentana. Satua bawak puniki sasampunne katilikin ngamolihang 7 wangun intrinsik sane jangkep. Unteng saking satua bawak sane mamurda Alpaka Nilar Sentana puniki inggih punika alpaka, lelintihan caritanyane nganggen lelintintihan maju. Satua bawak sane mamurda Alpaka Nilar Sentana puniki madue tiga soroh pragina inggih punika pragina dharma, pragina adharma lan pragina pewantu. Kawentenan pragina dharma ring satua bawak puniki wenten patpat inggih punika Gusti Ayu Cakrawati, Ajinne Gusti Ayu Cakrawati, Pan Arya lan Luh Aryani. Selanturnyane pragina adharma ring satua bawak puniki inggih punika Ngurah Arya taler pragina pewantu ring satua bawak puniki inggih punika Ibunne Gusti Ayu Cakrawati. Satua bawak puniki madaging tigang soroh rerawatan inggih punika rerawatan genah, galah lan kahanan.

Sasampunne katilikin satua bawak puniki madaging kalih rerawatan kemanten inggih punika rerawatan genah lan rerawatan galah. Wenten makudangkudang rerawatan genah sane kacaritayang ring satua bawak puniki minakadi ring RSU Mataram, ring gudang obat, Sasak, penambangan bus, pelabuhan Lembar, pelabuhan Padangbai lan jumahne Pan Arya. Selanturnyane rerawatan galah sane wenten ring satua bawak puniki inggih punika kalih masa, ri kala sore, wengi lan ri kala semengan. Genah linggih sang pangawi ring satua bawak puniki nglinggihang genah sane kaping tiga. Satua bawak puniki makehan nganggen basita paribasa inggih punika sesonggan. Kaping untat piteket ring satua bawak puniki mangda iraga stata eling tekening guru rupaka nenten alpaka.
Satua bawak sane kaping kalih inggih punika mamurda Jatu Karma, satua bawak puniki madue unteng tresna sujati. Lelintihan carita saking satua bawak puniki nganggen lelintihan campuran. Sasampunne satua bawak puniki katilikin, kapolihang kalih soroh pragina ring satua bawak puniki inggih punika pragina dharma lan pragina pewantu. Pragina dharma ring satua bawak puniki wenten tiga inggih punika titiang utawi Putu Widiada, Tan Swie Lian utawi Luh Siulan lan Made Widiary, taler pragina pewantunyane wenten kakeniang warga China, sersan Mudana, Tang Bing Yang lan Tan Bun Lie.

Kawentenan rerawatan sane wenten ring cerpen puniki wenten tigang soroh inggih punika rerawatan genah, galah lan kahanan. Wenten makudang-kudang rerawatan genah sane kacaritayang minakadi ring Lombok/Sasak, labuhan aji, penambangan Cakranegara, jumahne Putu Widiada, kamar tamu, ring natahe lan ring teras. Rerawatan galah ring satua bawak puniki kakeniang ri kala sore, wengi, semengan, jam roras tengai lan ring sasih kalima, taler rerawatan kahanan sane kacaritayang ri tepengan sedih lan bagia. Genah linggih sang pangawi ring satua bawak Jatu Karma puniki nglinggihang genah sane kapertama. Satua bawak Jatu Karma puniki makehan ngangge paribasa tetingkesan. Piteket sane prasida kaambil saking satua bawak puniki inggih punika ri kala iraga matetulung mangda kadasarin antuk tulus ikhlas sane sampun sinah kapungkuran pastika kawales antuk karma sne becik.

Satua bawak sane kaping tiga inggih punika mamurda Kehkeh Siap sane madue unteng kharmapala. Lelintihan caritanyane nganggen lelintihan maju. Ring satua bawak puniki taler madue tigang soroh pragina inggih punika pragina dharma, adharma lan pragina pewantu. Wenten patpat pragina dharma ring satua bawak puniki inggih punika Gusti Ayu Manik, Gusti Ayu Anggi lan polisi. Selanturnyane wenten makudang-kudang pragina adharma sane kacaritayang ring satua bawak puniki inggih punika Gusti Ketut Gudar/Modar, Made Dogolan, Iwan Saplar, Made Jimat lan Ketut Tubuhana. Selanturnyane pragina pewantu ring satua 
bawak puniki inggih punika dagang-dagang ring Pura Dalem, bebotoh, juru kembar, saya kalangan, Gusti Ayu Adhi lan kurenanne Iwan.

Rerawatan sane kacaritayang ring satua bawak puniki wenten rerawatan genah inggih punika kasinahang ring batan bingine, batan tiyinge, bet-bet di semane, Pura Dalem, kantor polisi, jumahne Iwan, jumahne Gusti Modar, di ampike, di paon lan di kamar mandi. Rerawatan galah sane kacaritayang inggih punika ri kala selidan, sanja lan jam kutus peteng. Rerawatan kahanan sane kacaritayang ring satua bawak puniki ri tepengan gedeg lan sedih. Genah linggih sang pangawinyane nglinggihang genah sane kaping tiga. Satua bawak Kehkeh Siap puniki makehan nganggen paribasa soroh sesawen. Piteket sane prasida kaambil ring satua bawak Kehkeh Siap puniki inggih punika iraga dados jadma yening nampi warisan mangda eling tekening swadharma dumun nenten ulap tekening warisan punika napi malih ngutang-ngutang.

Satua bawak sane kaping patpat mamurda Nyelsel Raga sane madue unteng semaya utawi janji. Lelintihan carita ring sajeroning satua bawak puniki nganggen lelintihan maju. Pragina sane wenten ring satua bawak Nyelsel Raga puniki wenten pragina dharma, adharma lan pewantu. Pragina dharmanyane wenten nemnem inggih punika Wayan Sudarsana, Luh Ginanti, pramugari, bapanne Wayan Sudarsana lan bapanne Luh Ginanti. Ring sajeroning pragina adharma wenten kalih inggih punika Made Gayatri lan Gusti Bagus Deko. Pragina kaping untat inggih punika pragina pewantu inggih punika reramane Made Gayatri, Putu Bagiasna, memene Wayan Sudarsana, memene Luh Ginanti, ida padanda, Bli Putu taler pimpinan lan pegawe Dinas.

$$
\text { Rerawatan genah sane }
$$

kacaritayang ring satua bawak Nyelsel Raga puniki inggih punika ring Kupang, bandara Kupang, ring tengah pesawat, Bandara Gusti Ngurah Rai, terminal, bale dangin lan umahne Luh Ginanti. Rerawatan galah sane kacaritayang inggih punika galah limang masa, amasa tengah, pitung tiban, aminggu, jam 12.00, ri kala sore, wengi, semengan, atengah jam, ibi sanja lan tigang rahina. Rerawatan kahanan ring satua bawak puniki ri tepengan sedih, inguh, galang lan bagia. Genah linggih sang pangawi ring satua bawak puniki nglinggihang genah sane kaping tiga. Paribasa sane kaanggen ring satua bawak Nyelsel Raga puniki makehan ngangge basita paribasa soroh tetingkesan. Kaping untat piteket sane prasida kaambil ring satua bawak sane mamurda Nyelsel Raga puniki mangda iraga dados jadma prasida satia tekening semaya utawi janji.

Satua bawak sane kaping untat inggih punika mamurda Pager Neda Tetanduran sane madue unteng satia utawi kesetiaan. Lelintihan carita sane kaanggen ring satua bawak puniki nganggen lelintihan maju. Ring sajeroning pragina sane wenten ring satua bawak puniki kapertama pragina dharma kakeniang antuk kalih pragina inggih punika Nyoman Jeladi lan adinne Nyoman Jeladi. Selanturnyane pragina adharma taler kakeniang kalih inggih punika Made Darmini lan Bagus Umbara. Pragina sane kaping untat inggih punika pragina pewantu sakadi truna-trunine, nyamanyamane Nyoman Jeladi lan timpaltimpalne Nyoman Jeladi.

Rerawatan sane wenten ring satua bawak puniki wenten rerawatan genah minakadi ring Jawi, jumahne Nyoman Jeladi lan ring bale banjar. Selanturnyane rerawatan galah inggih punika galah kalih masa lan ri kala jam pitu wengi, taler rerawatan kahanannyane ri tepengan demen utawi seneng lan ri kala sedih. Genah linggih sang pangawi ring satua bawak Pager Neda Tetanduran puniki nglinggihang genah sane kaping tiga. Paribasa sane makeh kaanggen ring satua bawak Pager Neda Tetanduran puniki inggih punika sesawangan. Kaping untat piteket sane prasida kaambil ring satua bawak puniki mangda iraga dados tunangan kukuh satia tekening janji lan pasangan taler yening iraga dados timpal mangda kukuh satia tekening pasawitran.

Pupulan satua bawak Kacunduk ring Besakih madaging 15 murda satua bawak, nanging panilik wantah nilikin 5 murda satua bawak minakadi Alpaka Nilar Sentana, Jatu Karma, Kehkeh Siap, Nyelsel Raga lan Pager Neda Tetanduran. Pikolih lan tetepasan tetilik basita paribasa 
sajeroning pupulan satua bawak Kacunduk ring Besakih pikardin I Gusti Gede Djelantik Santha katlatarang ring sor puniki.

Saking soang-soang murda satua bawak sane katilikin kapertama inggih punika mamurda Alpaka Nilar Sentana ngamolihang 4 soroh basita paribasa sakadi: sesonggan kapolihang 5 imba, raos ngempelin kapolihang 1 imba, sesawangan kapolihang 4 imba lan tetingkesan kapolihang 3 imba.

Saking satua bawak sane kaping kalih mamurda Jatu Karma kapolihang taler 4 soroh basita paribasa sakadi: tetingkesan kapolihang 1 imba, sesawen kapolihang 3 imba, sesonggan kapolihang 2 imba lan wewangsalan kapolihang 1 imba.

Satua bawak sane kaping tiga mamurda Kehkeh Siap kapolihang 6 soroh basita paribasa sakadi: sesawangan kapolihang 2 imba, bebladbadan kapolihang 1 imba, sesonggan kapolihang 5 imba, sesimbing kapolihang 4 imba, sesenggakan kapolihang 2 imba lan sesawen kapolihang 2 imba.

Satua bawak sane kaping patpat mamurda Nyelsel Raga sane ngamolihang 6 soroh basita paribasa sakadi: bebladbadan kapolihang 1 imba, sesawangan kapolihang 3 imba, tetingkesan kapolihang 4 imba, sesawen kapolihang 3 imba, wewangsalan kapolihang 1 imba lan sesonggan kapolihang 3 imba.

Satua bawak sane kaping untat inggih punika mamurda Pager $\mathrm{Neda}$ Tetanduran sane ngamolihang 3 soroh basita paribasa sakadi: sesawangan kapolihang 6 imba, bebladbadan 1 imba lan sesonggan kapolihang 3 imba.

Madasar antuk pikolih basita paribasa saking soang-soang murda satua bawak ring ajeng ngamolihang 9 soroh basita paribasa saking 13 soroh basita paribasa sane kaanggen sepat siku-siku. Akeh imbaimba basita paribasa ring ajeng kakeniang antuk 61 imba. Basita paribasa punika sakadi: bebladbadan kakeniang 3 imba, raos ngempelin kakeniang siki imba, sesawangan kakeniang 15 imba, patpat imba kakeniang ring sesimbing, sesenggakan kakeniang 2 imba, sesonggan kakeniang 18 imba, wewangsalan kakeniang antuk 2 imba, tetingkesan kakeniang antuk 8 imba lan sesawen taler kakeniang antuk 8 imba. Basita paribasa sane nenten kapolihang ring pupulan satua bawak Kacunduk ring besakih puniki inggih punika cecimpedan, sesapan, sloka lan peparikan.

Sinalih tunggil basita paribasa sane nenten wenten ring satua bawak Kacunduk ring Besakih inggih punika cecimpedan. Soroh cecimpedan nenten wenten ring kria sastra satua bawak saduaning sakadi teori cecimpedan punika ketah kaanggen pangulir budi olih anak alit-alit sane madaging pitaken-pitaken. Punika taler soroh peparikan sane arang kapanggihin ring sajeroning kria sastra modern sakadi satua bawak. Peparikan puniki ketah kapanggih ring sesolahan jejangeran sane marupa pantun tur katembangang sakadi tembang macepat. Indike puniki anut sakadi penampen Ginarsa (2009: 26), cutetnyane teges ring peparikan sampun megingsiran inggih punika pinaka tembang sane kaketus saking kekawin miwah katembangang nganggen tembang-tembang macepat. Yening anutin antuk murda-murda satua bawak Kacunduk ring Besakih sane katilikin dagingnyane makehan bebaosan sadinadina sane arang nganggen basita paribasa sakadi cecimpedan, sesapaan, sloka lan peparikan. Makehan bebaosan-bebaosan ring pupulan satua bawak Kacunduk ring Besakih puniki nganirgamayang parisolah jadma sane kasaihang antuk kahanan piranti, beburon miwah palemahan. Indike punika prasida nyutetang kawentenan basita paribasa sane makeh ring pupulan satua bawak Kacunduk ring Besakih puniki soroh sesonggan sane kasinahang antuk plekutus imba.

Ring ajeng sampun kasinahang makudang- kudang basita paribasa sane ring aab mangkin sampun arang kapanggihin parajanane sane nganggen basita paribasa ri kala mabebaosan. Basita paribasa ring ajeng kaanggen ri sajeroning kria sastra sane sumingkin arang kapanggih. Parajanane sakadi mangkin akehan seneng sareng parindikan sane to the point utawi nenten seneng sue-sue ri tatkala mabebaosan. Napi malih kasarengin antuk IImu Pengetahuan lan Teknologi (IPTEK) sane ngancan nglimbak ring kauripan. Basita paribasa pastika ngancan 
rered kawentenannyane saduaning mabebaosan nganggen basita paribasa nyinahang raos sane kantun makulit sane meweh karesepang tur wenten tata kramanyane. Pupulan satua bawak Kacunduk ring Besakih puniki prasida ngunggahang bebaosan-bebaosan nganggen basita paribasa sane mapikenoh ring kawentenan basita paribasa. Ring aab sane arang nganggen basita paribasa puniki, pupulan satua bawak Kacunduk ring Besakih puniki kacutetang madaging nem dasa siki imba basita paribasa sakadi pikolih ring ajeng.

Tetilik wangun intrinsik lan basita paribasa sajeroning karya sastra sujatinnyane sampun kalaksanayang olih panilik lianan. Mawit saking kawentenane punika panilik nyaihang pikolih lan tetepasan tetilik puniki sareng tetilik panilik lianan, sakadi: (1) tetilik A.A Raka Adi Suryawati "Seseleh Wangun Intrinsik lan Guna Sarat Paguron-guron ring Pupulan Cerpen Jro Lalung Ngutah Kakawian I.B.W Widiasa Keniten". Kapolihang pitu wangun intrinsik, dadosnyane pateh sareng tetilik puniki. Nanging ring sajeroning nelatarang soroh-soroh wangun intrinsik kasinahang durung jangkep pahannyane, (2) tetilik Gusti Ayu Era Aryanti "Seseleh Basita Paribasa lan Kawentenan Guna Sarat ring Daging Cakepan Drama Nang Kepod Pakardin Ketut Aryana". Kapolihang 9 soroh basita paribasa, dadosnyane pateh sareng tetilik puniki nanging pahan sorohnyane matiosan.

Yening kasaihang sareng tetilikan asoroh A. A Raka Adi Suryawati (2016) sane pateh nilikin wangun intrinsik prasida kawedarang pabinayannyane sakadi: (1) tetilik puniki nyinahang rerawatan sane tatas kakepah dados tiga inggih punika rerawatan genah, galah lan kahanan nanging ring tetilik Raka Adi Surya wantah nepasin indik rerawatan genah kemanten, (2) tetilik puniki nyinahang pragina sane kakepah dados tiga pahan inggih punika pragina dharma, adharma lan pewantu nanging ring tetilikan Raka Adi Surya nenten katatasang indik soroh pragina punika. Tetilikan sane kasaihang selanturnyane inggih punika tetilikan saking Gusti Ayu Era Aryanti (2015) sane pateh nilikin indik basita paribasa prasida kawedarang pabinayannyane sakadi: (1) tetilikan puniki mikolihang 9 soroh basita paribasa pateh sakadi tetilikan sane kasaihang nanging pahannyane matiosan, (2) tetilik puniki mikolihang 61 imba basita paribasa nanging ring tetilikan Gusti Ayu Era Aryati wantah mikolihang 40 imba basita paribasa. Punika pabinayannyane sasampunne kasaihang.

\section{PAMUPUT}

Manut pikolih lan tetepasan tetilik sane sampun katlatarang, data-data sane sampun kapolihang nganggen kramaning dokumentasi lan nganggen piranti kartu data. Antuk punika prasida kacutetang wangun intrinsik ring pupulan satua bawak Kacunduk ring Besakih pikardin I Gusti Gede Djelantik Santha wenten pitu sakadi: 1) unteng, (2) lelintihan carita, (3) pragina, (4) rerawatan, (5) genah linggih sang pangawi, (6) paribasa, lan (7) piteket. Wenten lelima satua bawak sane katilikin inggih punika satua bawak sane mamurda Alpaka Nilar Sentana, Jatu Karma, Kehkeh Siap, Nyelsel Raga lan Pager Neda Tetanduran. Saking lelima murda satua bawak sane katilikin makasami madue wangun intrinsik sane jangkep.

Basita paribasa sane wenten ring pupulan satua bawak Kacunduk ring Besakih puniki inggih punika wenten 9 soroh basita paribasa saking 13 soroh basita paribasa sane kaanggen ring sepat siku-siku. Taler indik akehnyane kakeniang antuk 61 imba. Basita paribasa punika sakadi: bebladbadan kakeniang 3 imba, raos ngempelin kakeniang siki imba, sesawangan kakeniang 15 imba, patpat imba kakeniang ring sesimbing, sesenggakan kakeniang 2 imba, sesonggan kakeniang 18 imba, wewangsalan kakeniang antuk 2 imba, tetingkesan kakeniang antuk 8 imba lan sesawen taler kakeniang antuk 8 imba. Dadosnyane, basita paribasa soroh sesonggan sane pinih akeh kapikolihang ring pupulan satua bawak Kacunduk ring Besakih. Basita paribasa sane nenten kapolihang ring pupulan satua bawak Kacunduk ring besakih puniki inggih punika cecimpedan, sesapan, sloka lan peparikan. 
Piteket ring sajeroning tetilik puniki inggih punika: (1) mangda prasida kaanggen dasar ring sajeroning pangresep indik wangun intrinsik ring sajeroning kria sastra utamannyane ring satua bawak, (2) mangda prasida dados dasar ri tatkala pacang nincapang kawagedan indik basita paribasa sane kasurat ring kria sastra utamannyane ring satua bawak, (3) majeng ring panilik lianan mangda sida nglimbakang malih indik daging tetilikan sane mapaiketan sareng wangun intrinsik lan basita paribasa ring kria sastra satua bawak.

\section{KAPUSTAKAAN}

Arikunto, Suharismi. 1998. Prosedur Penelitian Suatu Pendekatan Praktek. Jakarta: Rineka Cipta.

Djelantik Santha, I Gusti Gede. 2015. Kacunduk ring Besakih. Tabanan: Pustaka Ekspresi.

Gautama, Wayan Budha. 2007. Kesusastraan Bali. Cakepan Panuntun Malajahin Kesusastraan Bali. Gianyar: Paramita Surabaya.
Ginarsa, Ketut. 2009. Paribasa Bali. Singaraja: CV. Kayumas Agung

Merta, Putu Ngurah dkk. 2002. Mustikaning Basa Bali. Singaraja :Universitas Pendidikan Ganesha.

Putera, Zulfaisal. 2006. Penulisan Cerpen. Saatnya Menjadi Dewa. http://zulfaisalputera wordpress.com diakses pada tanggal 9 Januari 2017.

Simpen AB, Wayan. 1980. Basita Paribasa. Denpasar: Upada Sastra.

Suroto. 1989. Apresiasi Sastra Indonesia. Jakarta: Erlangga.

Tarigan, Henry Guntur. 1984. Prinsipprinsip Dasar Sastra. Bandung: Angkasa.

Tinggen, I Nengah. 1988. Aneka Rupa Paribasa Bali. Singaraja: Rikha Dewata

Warsito, R. H. 2012. Sisiologi: Sejarah, Teori dan Metodologi. Yogyakarta: Pustaka

Pelajar. 